Tempest, D. W. \& Smith, H. (1957). J. gen. Microbiol. 17, 739-749

\title{
The Effect of Metabolite Analogues on Growth of Bacillus anthracis in the Guinea pig and on the Formation of Virulence-determining Factors
}

\author{
By D. W. TEMPEST AND H. SMITH \\ Microbiological Research Establishment, Porton, Wiltshire
}

SUMMARY: Several metabolite analogues have been tested for their effect on the growth of Bacillus anthracis and its ability to synthesize toxin and capsular material during the terminal bacteraemic stage of anthrax in the guinea pig. 8-Azaguanine, 8 -azaxanthine and to a lesser degree, ethionine, $\alpha$-amino- $n$-butyric acid and $p$-fluorophenylalanine inhibited the in vivo growth of $B$. anthracis, whilst 2-thiouracil and pyridine 3-sulphonic acid selectively inhibited toxin production. Inhibition of capsule formation was at no time convincingly demonstrated. The annulment of these inhibitions by mixture of the analogues with appropriate metabolites gives evidence that hypoxanthine, adenine, methionine, alanine, phenylalanine and tryptophan are involved in growth in vivo and pyrimidines and nicotinamide in toxin synthesis.

To multiply in the tissues of a host and to produce disease a pathogen must form products in addition to new cell material: it must elaborate aggressins to combat the defence mechanisms of the host, and form toxic products or processes responsible for the symptoms of the disease. When growing in the guinea pig, Bacillus anthracis forms at least two aggressins, capsular polyglutamic acid and a specific oedema-producing toxin which later in the course of the disease acts to kill the host (Zwartouw \& Smith, 1956; Smith, Zwartouw \& Harris-Smith, 1956; Smith, Keppie \& Stanley, 1955; Smith, Tempest, Stanley, Gallop \& Harris-Smith, 1956). As part of an attempt to study the metabolism of $B$. anthracis when multiplying in an infected animal and producing these virulence factors, the utilization of amino acids by $\boldsymbol{B}$. anthracis when growing in the blood stream of a guinea pig was studied as described in the previous paper (Smith \& Tempest, 1957). These results were measurements of amino acid uptake and gave no indication about which amino acids were essential for the growth of the parasite and production of virulence factors. The present paper describes experiments to investigate the more specific role of various amino acids in this respect and the studies have been extended to include purines, pyrimidines and vitamins.

In experiments in vitro, the importance of each metabolite could be assessed by altering the composition of the environment so as to render it deficient in that compound. It was impossible to carry out this experimental arrangement when dealing with the metabolism of Bacillus anthracis in the tissues of a guinea pig. We therefore attempted to block the utilization of individual metabolites by injecting metabolite analogues into guinea pigs and to study the subsequent effects upon the growth of $B$. anthracis and on the synthesis of virulence-determining factors. 
In the $10 \mathrm{hr}$. before death from anthrax in the guinea pig, capsulated Bacillus anthracis organisms proliferate in the blood stream and produce toxin which accumulates in the plasma. It has been pointed out (Keppie, Smith \& Harris-Smith, 1955; Smith \& Tempest, 1957) that in this final phase of the disease the growth rate of $B$. anthracis in the blood is remarkably constant, and it will be shown that this also applies to the rate of accumulation of free toxin. We had, therefore, a system suitable to study the effect of metabolite analogues on growth, capsulation and toxin production by $B$. anthracis. It was during this final $10 \mathrm{hr}$. of the disease that all experiments were carried out. Relatively large quantities of the analogues were injected directly into the blood stream and observations were made over relatively short time intervals. In this way, possible effects of the analogues other than a direct effect on the metabolism of the parasite were minimized. When the injection of any metabolite analogue produced a noticeable effect on the growth of $B$. anthracis or on the synthesis of virulence factors, a search was made for the metabolite which would annul this effect. Until recently, when the anthrax toxin was produced in vitro (Harris-Smith, Smith \& Keppie, 1957) and the major part of this work completed, experiments in vivo were essential for metabolic studies on the synthesis of the anthrax toxin.

\section{METHODS}

Samples of metabolites and metabolite analogues. Alloisoleucine, $\alpha$-amino-isopentane-sulphonic acid, $\alpha$-amino-isobutane-sulphonic acid, methionine sulphoxide, S-methyl cysteine, $\alpha$-methylserine, N-ethylglycine, aminomethane sulphonic acid, $\alpha$-methyl glutamic acid, aspartyl-hydroxylamine, glutamyl hydrazine, and canavanine were kindly prepared by our colleague Mr S. Lovatt, to whom we are indebted. The remainder of the metabolites and their analogues were commercial products supplied by L. Light and Co., British Drug Houses Ltd., and Nutritional Biochemical Co. Most of the analogues were examined for purity either by paper chromatography (amino acid analogues) or by ionophoresis (purine and pyrimidine analogues) as described below, and were found to be free from obvious impurities.

All samples of amino acids used for annulment of inhibition experiments were free from ninhydrin-reacting impurities when examined by paper chromatography in butanol + acetic acid + water (125:30:125). Samples of purines and pyrimidines used for annulment experiments were free from ultravioletabsorbing impurities when examined after ionophoresis on paper in butyrate buffer at $\mathrm{pH} \mathrm{3.2}$ as described by Wade \& Morgan (1954). Xanthine was the only exception; it contained small amounts of guanine, but no adenine or hypoxanthine. A sample of xanthine purified by using Dowex 50 at $\mathrm{pH} 3$ was used in some of these experiments.

Selection of animals for injection. Guinea pigs $(650 \pm 50 \mathrm{~g}$.) were infected with Bacillus anthracis, strain N.P. On the third day, the terminal bacteraemia was detected and followed by using stained blood films from the ears as described by Keppie et al. (1955). Animals having bacterial counts of 
$0 \cdot 5-5 \times 10^{6}$ chains $/ \mathrm{ml}$. blood were selected for experiment. In the normal course such animals would die 7·5-11 hr. later (Keppie et al. 1955).

Dosage of metabolite analogues. A single large dose (250-1000 mg.) of metabolite analogue was injected. Sometimes the dose was limited by the availability of the analogue or by an immediate toxic effect on the host. Any delayed toxic effect, due to the large dose of an analogue acting alone or in combination with the later stages of the anthrax syndrome, did not interfere with the experiment as long as there was time to take samples for observations before the guinea pig died.

Injection of analogue. When soluble in saline, the metabolite analogue was dissolved in $20 \mathrm{ml}$. and the $\mathrm{pH}$ value adjusted to $7 \cdot 5 \pm 0 \cdot 5$. Half this solution was injected intravenously into an exposed vein of the hind leg and the remainder intraperitoneally. When the metabolite analogue was only partially soluble in saline, it was suspended in $20 \mathrm{ml}$., warmed to $60^{\circ}$, adjusted to $\mathrm{pH} 7 \cdot 5 \pm 0.5$ and allowed to cool. Ten $\mathrm{ml}$. of the saturated solution were removed, filtered through Whatman no. 1 paper and injected intravenously. A suspension of the undissolved solid in the remaining solution was injected intraperitoneally.

Determination of the increase in bacterial content of the blood. At various time intervals blood films from the ear were prepared in the manner described by Keppie et al. (1955). The average number of chains/field was related to the number of chains/ml. blood (see Keppie et al. 1955) and was subject to a maximum error of $\pm 50 \%$. In all but a few cases, when the metabolite analogue was injected the average chain length remained approximately the same as in the normal bacteraemia. In those cases when the chain length varied more than twofold from the normal average, appropriate correction was made in the recorded bacterial count.

Collection and assay of toxin. Blood $(2 \mathrm{ml}$.) was obtained by cardiac puncture and mixed with ice-cold distilled water $(6 \mathrm{ml}$.). The lysed blood was centrifuged $(1500 \mathrm{~g}$ for $30 \mathrm{~min})$ at $4^{\circ}$ and the supernatant fluid filtered free of bacteria through Millipore filters. Twofold serial dilutions $(\mathbf{0 . 2} \mathrm{ml}$.) were injected intradermally into a depilated guinea pig. After $20 \mathrm{hr}$. the highest dilution (relating to the original volume of blood) which just produced a detectable oedematous reaction was noted; the strength of the toxin was quoted in units as the reciprocal of this dilution. The assay detected twofold differences in toxin. When intravenous administration of a metabolite analogue apparently decreased toxin production, any possibility that the analogue interfered with the assay was excluded by testing a mixture of normal toxin + analogue.

Observation of bacterial capsules. Capsules were evident on the organisms in blood smears stained by methylene blue (Macfadyean reaction). When the capsule appeared diminished or absent, observations were made on wet films containing nigrosine or Indian ink. 


\section{RESULTS \\ Normal rate of bacterial increase and free toxin appearance in the blood during the terminal bacteraemia}

Since we were interested in assessing the changes brought about in the blood of guinea pigs infected with Bacillus anthracis by the injection of metabolite analogues, it was important to establish that the normal degrees of bacterial increase and free toxin appearance in the blood were reasonably constant from animal to animal and not unduly affected by the other tissues of the body.

The rate of bacterial increase. Keppie et al. (1955) showed that the count of bacterial chains in the blood increased regularly throughout the $10 \mathrm{hr}$. which preceded death from anthrax and that this increase was remarkably constant from animal to animal. Any change in this regular pattern brought about by injection of a metabolite analogue might reasonably be ascribed to the direct effect of the analogue on the growth of the infecting bacteria since the majority of the organisms are located in the blood during this late stage of the disease (see Table 2 of Keppie et al. 1955).

The rate of accumulation of toxin in the blood. Samples (23) of blood taken from a number (14) of guinea pigs at different stages of the final bacteraemia were assayed for toxin. A plot of the toxin content against the number of chains of bacilli/ml. blood showed that the toxin content rose regularly with the bacterial count and was reasonably constant from animal to animal (see Figs. 5 and 6).

The amount of toxin detected here is that produced by the infecting bacteria over and above the amount which is adsorbed by the host's tissues or destroyed (or toxoided) by the growing bacteria. There is evidence which suggests that the amount of undetected toxin was considerable. Thus (i) the amount of circulating toxin per organism decreased as the bacteraemia progressed; this is indicated by the low slope of the normal toxin/bacterial count plot; (ii) fol- . lowing intravenous injection of toxin into a non-infected guinea pig the amount of circulating toxin decreased fourfold every hr.; (iii) when an amount of antitoxin capable of neutralizing more than 10 times the total amount of free toxin normally detectable at the end of the bacteraemia was injected early in the bacteraemia, this did not prevent unneutralized toxin from being found at the somewhat delayed death of the animal. Nevertheless, the rate of free toxin accumulation in the blood reflects that produced by the organism and any significant change in overall production will reflect itself on the amount of free toxin.

\section{Preliminary survey of metabolite analogues}

Fifty-seven metabolite analogues were screened for their effect on the growth, toxin production and capsulation of Bacillus anthracis. After injection of the analogue, blood films were examined at intervals to determine the extent of capsulation and the rate of bacterial increase but the toxin content of the blood was determined only at the end of the bacteraemia. The results 
are given in Table 1. Only a few of the analogues produced significant differences from the normal observations; the behaviour of these compounds was investigated in some detail.

Table 1. The effect of metabolite analogues on growth of Bacillus anthracis in the guinea pig and on the synthesis of virulence-determining factors

$\begin{array}{llll} & \text { Dose } & \text { Dose } \\ \text { Metabolite analogues } & \text { (mg.) } & \text { Metabolite analogues } & \text { (mg.) }\end{array}$

Metabolite analogues which were ineffective in vivo $\dagger$

\begin{tabular}{|c|c|}
\hline Norleucine & 1000 \\
\hline Norvaline & 1000 \\
\hline D-Norvaline & 1000 \\
\hline Allo isoleucine & 1000 \\
\hline$\alpha$-Amino-isopentane sulphonic acid & 250* \\
\hline$\alpha$-Amino-isobutane sulphonic acid & 500 \\
\hline Isovaline & 1000 \\
\hline Methioninol & 250* \\
\hline Methionine sulphoxide & 1000 \\
\hline S-Methyl cysteine & 1000 \\
\hline D-Tryptophan & 500 \\
\hline Acetyltryptophan & 1000 \\
\hline Monochloro-l-tyrosine & 1000 \\
\hline 3-Amino tyrosine & 500 \\
\hline$p$-Chlorophenylalanine & 500 \\
\hline$o$-Fluorophenylalanine & 500 \\
\hline$\beta$-2-Thienylalanine & 500 \\
\hline D-Threonine & 750 \\
\hline Allo threonine & 1000 \\
\hline$\alpha$-Methylserine & 500 \\
\hline Serinol & 500 \\
\hline$\alpha$-Amino-isobutyri & 1000 \\
\hline$\gamma$-Amino-butyric acid & 1000 \\
\hline
\end{tabular}

$\begin{array}{lc}\text { N-Ethylglycine } & 1000 \\ \text { Amino methane sulphonic acid } & 250^{*} \\ \text { a-Methyl glutamic acid } & 1000 \\ \beta \text {-Hydroxy glutamic acid } & 500 \\ \text { Ethyl } \gamma \text {-l-glutamate } & 1000 \\ \text { Aspartyl hydroxylamine } & 100 \\ \text { Canavanine } & 500 \\ \gamma \text {-Glutamyl hydrazine } & 150^{*} \\ \text { Methionine sulphone } & 2000 \\ \text { 8-Aza-adenine } & \mathbf{2 5 0} \\ \text { 2:6-Diaminopurine } & 500 \\ \text { 6-Mercaptopurine } & 500 \\ \text { 2-Thio-6-oxypurine } & 500 \\ \text { 8-Chloroxanthine } & 500 \\ \text { Benzimidazole } & 300 \\ \text { 6-Nitrobenzimidazole } & 500 \\ \text { Thiothymine } & 500 \\ \text { Isocytosine } & 500 \\ p \text {-Aminosalicyclic acid } & 500 \\ \text {-Aminoacetophenone } & 250 \\ \text { 6-Chloronicotinamide } & 500 \\ \text { Isonicotinyl hydrazide } & 100^{*} \\ \text { D-Pantothenylalcohol } & 1000 \\ \text { 2-Amino thiazole } & 100^{*}\end{array}$

Metabolite analogues which decrease growth in vivo

$\begin{array}{lllr}\text { 8-Azaguanine } & 250 & \text { Ethionine } & 500 \\ \text { 8-Azaxanthine } & 250 & p \text {-Fluorophenylalanine } & 500 \\ & & \alpha \text {-Amino- } n \text {-butyric acid } & 1000\end{array}$

Metabolite analogues which decrease toxin synthesis in vivo

$\begin{array}{lc}\text { Pyridine 3-sulphonic acid } & 1000 \\ \text { 3-Acetylpyridine } & 500^{*}\end{array}$

2-Thiouracil 500

4-Methyl 2-thiouracil $\quad 500$

2-Thio-6-amino uracil $\quad 500$

5-Bromo-uracil $\quad 500$

* Toxic to guineapigs at higher concentrations.

$\dagger$ Injection of these analogues did not produce a rate of increase of bacterial chains in the blood significantly different from the normal (Fig. 1). The toxin amounts present at the end of the bacteraemia were not less than 64 and not more than 256 units (see p. 741). There was no significant diminution in capsule.

Analogues which decreased the in vivo growth rate of Bacillus anthracis

8-Azaguanine. This was the most effective growth inhibitor examined in vivo. Injection of $250 \mathrm{mg}$. brought about complete bacteriostasis for a considerable time and $50 \mathrm{mg}$. drastically decreased the rate of multiplication 
(Fig. 1). Simultaneous injection of hypoxanthine annulled the effect of azaguanine; guanine was much less effective in this respect. When quantities of guanine equimolecular to that of the analogue were injected only a small decrease in the inhibition took place; this was increased by raising the proportion of metabolite to analogue. In these annulment experiments adenine replaced hypoxanthine, and xanthine behaved like guanine.

8-Azaxanthine. Experiments showed that the effect was similar to that described for azaguanine.

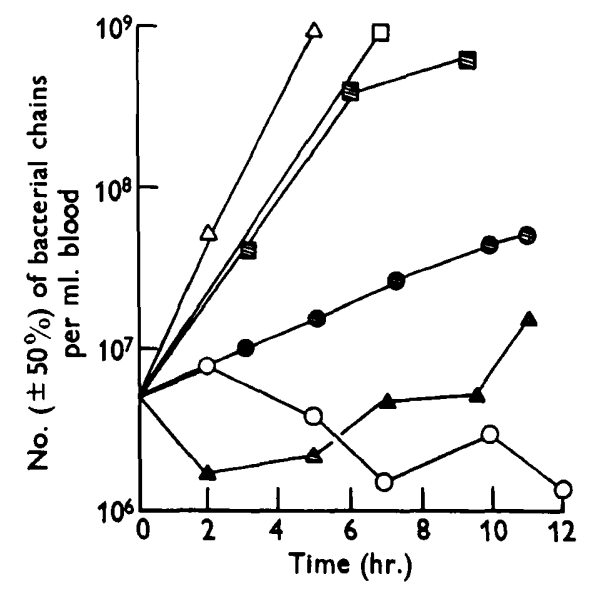

Fig. 1

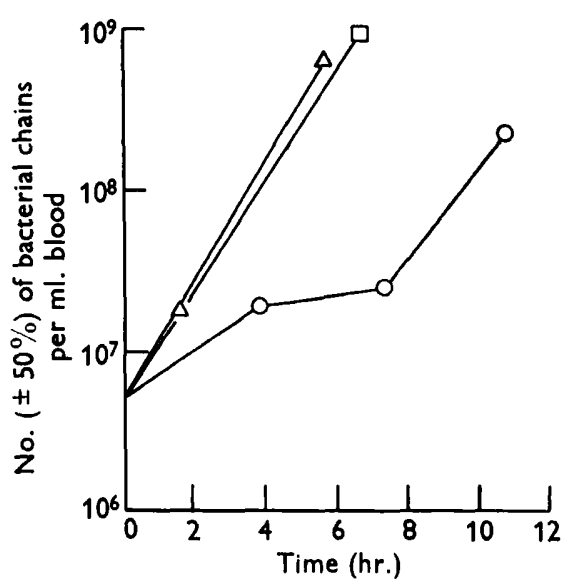

Fig. 2

Fig. 1. The inhibition of in vivo growth of Bacillus anthracis by 8-azaguanine and the reversal of this effect by purines. $\square$, Normal untreated animal; $O$, 8-azaguanine (250 mg.); $\triangle$, 8-azaguanine (250 mg.) + hypoxanthine (250 mg.); $\Delta$, 8-azaguanine (250 mg.) + guanine (250 mg.); $\$$, 8-azaguanine (50 mg.); $\mathbb{\$}, 8$-azaguanine (50 mg.) +guanine $(1000 \mathrm{mg}$.). The graphs shown represent the data of the most representative experiment of two to six similar experiments.

Fig. 2. The inhibition of in vivo growth of Bacillus anthracis by ethionine and its annulment by mixture with methionine. $\square$, Normal untreated animal; $O$, ethionine (500 mg.); $\triangle$, ethionine (500 mg.) + methionine (500 mg.). The graphs shown represent the data of the most representative experiment of two to five similar experiments.

Ethionine. The results given in Fig. 2 show that injection of ethionine (500 mg.) decreased the growth rate, and that this effect was annulled by simultaneous injection of methionine. Leucine, isoleucine, valine, tryptophan, tyrosine, phenylalanine, glutamic acid, aspartic acid, proline, alanine, glycine, serine, arginine, lysine, threonine, cysteine and histidine, given in groups of two or three compounds, did not annul the effect.

$\alpha$-Amino $\mathrm{n}$-butyric acid. Injection of $\alpha$-amino $n$-butyric acid (1000 mg.) decreased the growth rate, and this effect was annulled by simultaneous injection of alanine (Fig. B). Injection of leucine, isoleucine, valine, tryptophan, tyrosine, phenylalanine, glutamic acid, aspartic acid, proline, glycine, threonine, serine, arginine, lysine, methionine, cysteine or histidine did not annul the effect. 


\section{Antimetabolites and virulence of $\mathrm{B}$. anthracis}

$p$-Fluorophenylalanine. The inhibitory effect of this analogue $(500 \mathrm{mg}$.) was not annulled by simultaneous injection of leucine, isoleucine, valine, glutamic acid, aspartic acid, proline, methionine, histidine, cysteine, alanine, glycine, serine, threonine, arginine, lysine or tyrosine. Phenylalanine and tryptophan partially annulled the effect but only produced complete annulment when injected together (Fig. 4).

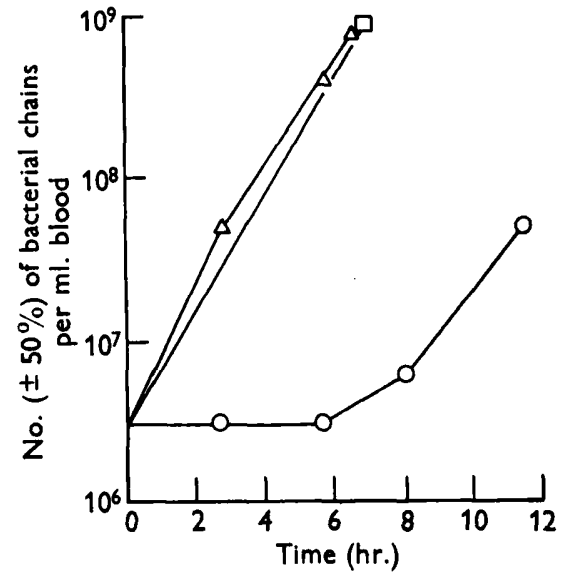

Fig. 3

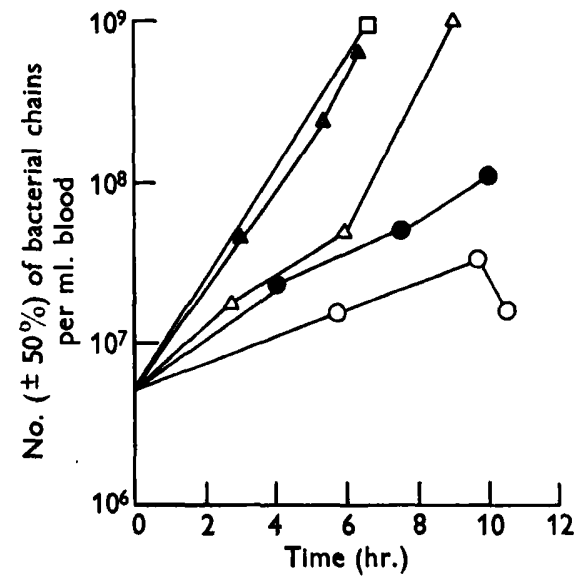

Fig. 4

Fig. 3. The inhibition of in vivo growth of Bacillus anthracis by $\alpha$-amino- $n$-butyric acid and its annulment by mixture with alanine. $\square$, Normal untreated animal; $0, \alpha$-amino- $n$ butyric acid (1000 mg.); $\triangle, \alpha$-amino- $n$-butyric acid (1000 mg.) +alanine (1000 mg.); The graphs shown represent the data of the most representative experiment of two to five similar experiments.

Fig. 4. The inhibition of in vivo growth of Bacillus anthracis by $p$-fluorophenylalanine and its annulment by mixture with phenylalanine +tryptophan. $\square$, Normal untreated animal; $O, p$-fluorophenylalanine $(500 \mathrm{mg}$ ); $\Delta, p$-fluorophenylalanine (500 mg.) + phenylalanine (500 mg.); $\$ p$-fluorophenylalanine (500 mg.) + tryptophan (500 mg.); 今, $p$-fluorophenylalanine (500 mg.) + phenylalanine (250 mg.) + tryptophan (250 mg.). The graphs shown represent the data of the most representative experiment of two to five similar experiments.

In all these cases where the growth rate was decreased by injection of a metabolite analogue the total toxin production was also decreased. In the case of 8-azaguanine the amount of free toxin in the blood corresponded with the number of bacterial chains/ml. according to the normal toxin/growth relationship shown in Fig. 5 throughout the whole course of the inhibition. In some cases, however, there was evidence that the rate of toxin production/organism was not decreased to the same degree as the rate of growth. This difference was not apparent in the immediate period following injection of the metabolite analogue but only became evident in the later samples taken at the death of the animal. Thus, injection of ethionine, $p$-fluorophenylalanine and $\alpha$-amino$n$-butyric acid into different animals produced the following increased toxin content of the blood at the indicated bacterial count (chains/ml.); the normal toxin content in units (see page 741) which corresponds to the bacterial count in 
the uninhibited system is given in brackets: ethionine, 48 (12) at $4.5 \times 10^{7}$, $96(16)$ at $7.5 \times 10^{7}, 64(24)$ at $9.0 \times 10^{7} ; p$-fluorophenylalanine $32(12)$ at $4.5 \times 10^{7}, 48(24)$ at $9.0 \times 10^{7}, 64(32)$ at $1.5 \times 10^{8}, \alpha$-amino- $n$-butyric acid $48(12)$ at $4.5 \times 10^{7}, 128(16)$ at $7.5 \times 10^{7}, 96(48)$ at $3.9 \times 10^{8}$. It seems therefore that growth and toxin production can be divorced from one another; this is amply confirmed by the results shown in the next section.

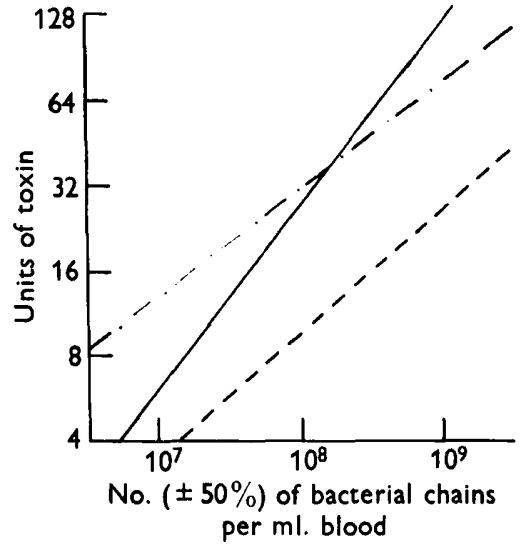

Fig. 5

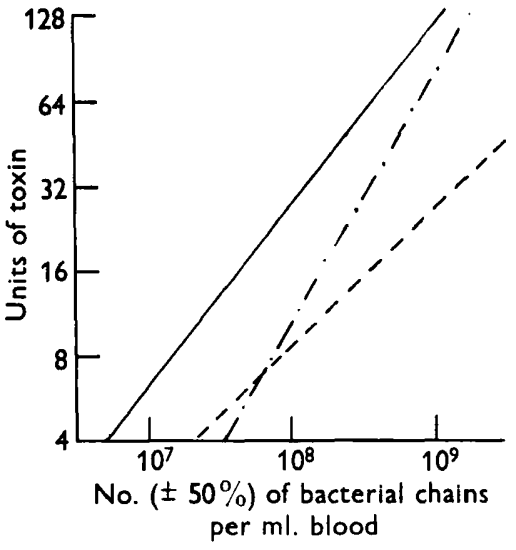

Fig. 6

Fig. 5. The inhibition of toxin production by 2-thiouracil and its annulment by uracil. - Growth/toxin curve in untreated animals. $y=0.62 x-0 \cdot 46 \pm 0 \cdot 27$ (fiducial limits for $P=95 \%$ ). - - , Growth/toxin curve in animals treated with $500 \mathrm{mg}$. 2-thiouracil. $y=0 \cdot 43 x-2 \cdot 45 \pm 0 \cdot 46$ (fiducial limits for $P=95 \%$ ). - - - . - Growth/toxin curve in animals treated with 2-thiouracil (500 mg.) + uracil (500 mg.) $y=0 \cdot 37 x-1 \cdot 49 \pm 0 \cdot 48$ (fiducial limits for $\boldsymbol{P}=95 \%$ ).

Fig. 6. The inhibition of toxin production by pyridine 3-sulphonic acid and the effect of simultaneous injection of nicotinamide. - Growth/toxin curve in untreated animals. $y=0.62 x-0.46 \pm 0.27$ (fiducial limits for $P=95 \%$ ). --- , Growth/toxin curve in animals treated with pyridine 3-sulphonic acid. $y=0 \cdot 48 x-2 \cdot 88 \pm 0 \cdot 45$ (fiducial limits for $P=95 \%$ ). - - - Growth/toxin curve in animals treated with pyridine 3sulphonic acid (1000 mg.) + nicotinamide (500 mg.). $y=0.92 x-6 \cdot 42 \pm 0 \cdot 47$ (fiducial limits for $P=95 \%$ ). N.B. Admixture with nicotinamide (1000 mg.) was too toxic to the guinea pig for observation to be made.

\section{Metabolite analogues which decrease the rate of toxin production without materially decreasing growth rate or capsule production}

2-Thiouracil. Samples (24) of blood from animals (18) which had been injected with thiouracil $(500 \mathrm{mg}$.) were taken at different stages in the terminal bacteraemia. The mean rate of increase of bacteria (log number of chains $/ \mathrm{ml}$. blood $/ \mathrm{hr}$.) in these thiouracil-treated animals 0.33 (s.E. 0.02), was not significantly different from the normal rate 0.34 (s.E. 0.01). However, the curve of the toxin content of the blood plotted against number of chains $/ \mathrm{ml}$. was significantly different from that of the normal curve (Fig. 5). Toxin production had been inhibited although the rate of growth of the Bacillus anthracis was normal. Simultaneous injection of uracil $(500 \mathrm{mg}$.) with thiouracil (500 mg.) increased toxin production (see Fig. 5) to normal values 
although the slope of toxin increase with bacterial count was somewhat lower than the normal slope. In smaller scale experiments cytosine, thymine or orotic acid annulled the effect of 2-thiouracil.

4-Methyl-2-thiouracil, 2-thio-6-amino-uracil and 5-bromo-uracil were not examined further than in the preliminary tests since it seemed likely that their mode of action would have been the same as 2-thiouracil.

Pyridine 3-sulphonic acid. Pyridine 3-sulphonic acid (1000 mg.) was injected into a number of animals (10) and samples (15) of blood taken for analysis as described for the experiments with thiouracil. Again, the rate of increase of bacteria (log number of chains in the blood/hr.) in the treated animals (0.32 s.E. 0.02) was not significantly different from the normal rate but toxin formation was inhibited (Fig. 6). Simultaneous injection of nicotinamide (500 mg.) partially annulled the effect of pyridine 3-sulphonic acid in the later stages of the bacteraemia (see Fig. 6), but simultaneous injection of uracil did not.

3-Acetylpyridine. This metabolite analogue was rapidly toxic to test animals at doses in excess of $\mathbf{5 0 0} \mathbf{m g}$. At this dose, a small but significant decrease in amount of free toxin/organism occurred, but since the rate of growth of the organism was much stimulated by the injection of this metabolite analogue (this corrects the statement of Tempest \& Smith, 1956), the results are difficult to assess.

The analogues which affected toxin production did not significantly affect the capsulation of the organisms.

\section{DISCUSSION}

Some success has attended the use of metabolite analogues to investigate the amino acids and other nutrilites important in the in vivo growth and toxin production of Bacillus anthracis. There were difficulties attending these experiments, thus, the trapping of growing organisms in the reticulo-endothelial system, the growth of $\boldsymbol{B}$. anthracis in chains rather than single organisms, the possible production of toxin by organisms in sites other than the blood, the unknown rates of adsorption and detoxification of toxin by the tissues of an infected guinea pig and, above all, the effect of the metabolite analogue on all these factors. This allowed only a semi-quantitative treatment of results. However, it was shown that some metabolite analogues did decrease growth in vivo and concurrently the toxin production of $\boldsymbol{B}$. anthracis, while others suppressed toxin production without interfering with growth. The annulment of these effects by corresponding metabolites serves as evidence for the specific use of these metabolites by $B$. anthracis in the appropriate metabolic processes in vivo.

8-Azaguanine was the most effective growth inhibitor. The specificity of the annulment of this inhibition is somewhat at variance with the results of workers who used other organisms (Creaser, 1956) especially in that guanine is less able to counteract the effect of 8-azaguanine with Bacillus anthracis in guinea pigs, than is adenine and hypoxanthine. It is possible that the 
behaviour of guanine and of xanthine is due to their low solubility in the tissue fluids.

It appears from the annulment of the effects of ethionine, $p$-fluorophenylalanine and $\alpha$-amino- $n$-butyric acid that the unimpaired metabolism of methionine, phenylalanine, alanine and tryptophan are essential for in vivo growth of Bacillus anthracis. All these amino acids are present in adequate concentrations in the free amino acid pool of the guinea pig plasma but only tryptophan is taken up by $B$. anthracis in appreciable quantities (Smith \& Tempest, 1957).

The inhibition of toxin synthesis by 2-thiouracil or pyridine 8-sulphonic acid and the annulment of their effects by various pyrimidines or nicotinamide, respectively, suggests that the latter compounds are involved in the processes leading to toxin production. It is possible that the two metabolite analogues inhibit the production of only one of the two factors in the anthrax toxin (Smith et al. 1956) but this point has not been investigated.

Only one of the metabolite analogues examined, namely methionine sulphone, produced a noticeable effect on capsulation. This was apparent as a loss in staining property but examination of the organisms in wet Indian ink preparations showed the general structure of the capsule to be intact. It is of interest that Thorne (1956) showed that alanine and phenylalanine (analogues of which limit growth in vivo) are intimately connected with capsule synthesis in closely related organisms.

Our thanks are due to our colleagues Dr J. Keppie and Mrs Patricia W. HarrisSmith for help with the animal experiments and to Mr G. S. Grace for skilled technical assistance.

\section{REFERENCES}

Creaser, E. H. (1956). The assimilation of amino acids by bacteria. 22. The effect of 8-azaguanine upon enzyme formation in Staphylococcus aureus. Biochem. J. 64, 539 .

Harris-Smith, P. W., Smith, H. \& Keppie, J. (1957). Production in vitro of the anthrax toxin previously recognized in vivo. J. gen. Microbiol. 16, viii.

Keppie, J., Smith, H. \& Harris-Smith, P. W. (1955). The chemical basis of the virulence of Bacillus anthracis. III. The role of the terminal bacteraemia in death of animals from anthrax. Brit. J. exp. Path. 36, 315.

Smith, H., Keppie, J. \& Stanley, J. L. (1955). The chemical basis of the virulence of Bacillus anthracis. V. The specific toxin produced by B. anthracis in vivo. Brit. J. exp. Path. 36, 460.

Smith, H. \& Tempest, D. W. (1957). The uptake of amino acids during the terminal bacteraemia in guinea pigs infected with Bacillus anthracis. J. gen. Microbiol. $17,731$.

Smith, H., Tempest, D. W., Stanley, J. L., Gallop, R. C. \& Harris-Smith, P. W. (1956). The chemical basis of the virulence of Bacillus anthracis. VII. Two components of the anthrax toxin: their relationship to known immunising aggressins. Brit. J. exp. Path. 37, 263.

Smith, H., Zwartouw, H. T. \& Harris-Smith, P. W. (1956). The chemical basis of the virulence of Bacillus anthracis. VIII. Fractionation of the intracellular material of Bacillus anthracis. Brit. J. exp. Path. 37, 361. 
Tempest, D. W. \& Smith, H. (1956). The effect of metabolite analogues on some virulence factors of Bacillus anthracis in the guinea pig. J. gen. Microbiol. 15, v.

TrorNe, C. G. (1956). Capsule formation and glutamyl polypeptide synthesis by Bacillus anthracis and Bacillus subtilis. In Bacterial Anatomy. Symp. Soc. gen. Microbiol. 6, 68.

WADE, H. E. \& Morgan, D. M. (1954). The analysis of adenosine triphosphate and adenosine diphosphate preparations by paper ionophoresis. Biochem. J. 56, 41.

Zwartouw, H. T. \& SMITh, H. (1956). Polyglutamic acid from Bacillus anthracis grown in vivo: Structure and aggressin activity. Biochem. J. 63, 437.

(Received 4 July 1957) 